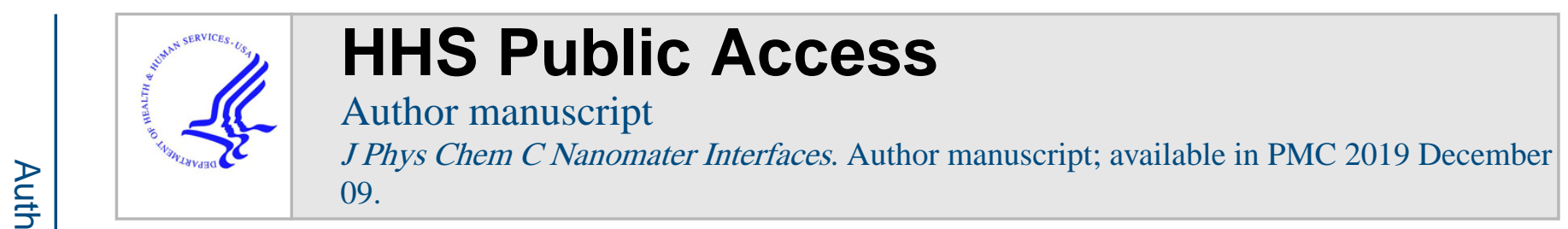

Published in final edited form as:

J Phys Chem C Nanomater Interfaces. 2019 ; 123(15): 9813-9823. doi:10.1021/acs.jpcc.9b00297.

\title{
Dye Encapsulation in Fluorescent Core-Shell Silica Nanoparticles as Probed by Fluorescence Correlation Spectroscopy
}

\author{
Ferdinand F. E. Kohle ${ }^{\dagger, \ddagger}$, Joshua A. Hinckley ${ }^{\dagger, \ddagger}$, Ulrich B. Wiesner ${ }^{\dagger,}{ }^{\star}$ \\ †. Materials Science and Engineering, Cornell University, Ithaca, NY 14853 \\ ¥. Chemistry and Chemical Biology, Cornell University, Ithaca, NY 14853
}

\begin{abstract}
Synthetic advances in the formation of ultrasmall $(<10 \mathrm{~nm})$ fluorescent poly(ethylene glycol)coated (PEGylated) core-shell silica nanoparticles (SNPs), enabling improved particle size and surface chemical property control have led to successful clinical translation of SNPs as diagnostic probes in oncology. Despite the success of such probes, details of the dye incorporation and resulting silica architecture are still poorly understood. Here, we employ afterpulse-corrected fluorescence correlation spectroscopy (FCS) to monitor fast fluorescence fluctuations (lag times $<10^{-5} \mathrm{~s}$ ) of the negatively charged cyanine dye Cy5 as a probe to study such details for dye encapsulation in $5 \mathrm{~nm}$ silica cores of PEGylated core-shell SNPs (C dots). Upon deposition of additional silica shells over the silica core we find that the amplitude of photo-induced cis-trans isomerization decreases, suggesting that the Cy5 dyes are located near or on the surface of the original SNP cores. In combination with time correlated fluorescence decay measurements we deduce radiative and non-radiative rates of the $\mathrm{Cy} 5$ dye in these particles. Results demonstrate that FCS is a well-suited tool to investigate aspects of the photophysics of fluorescent nanoparticles, and that conformational changes of cyanine dyes like Cy5 are excellent indicators for the local dye environment within ultrasmall SNPs.
\end{abstract}

\section{Graphical Abstract}

\footnotetext{
*Department of Materials Science and Engineering, Cornell University, 330 Bard Hall, Ithaca, NY 14853. ubw1@ cornell.edu Fax: 607-255-2365.

SUPPORTING INFORMATION

Supporting Information Available: The supporting information contains additional gel permeation chromatography (GPC) elugrams and optical spectra of Cy5 core particles, transmission electron microscopy (TEM) images of Cy5 core and core-shell particles and particle size distributions, fluorescence lifetime and quantum yield measurements, as well as tabulated fitting parameters of fluorescence correlation spectroscopy measurements and fluorescence lifetime measurements.

This material is available free of charge via the Internet at http://pubs.acs.org.
} 


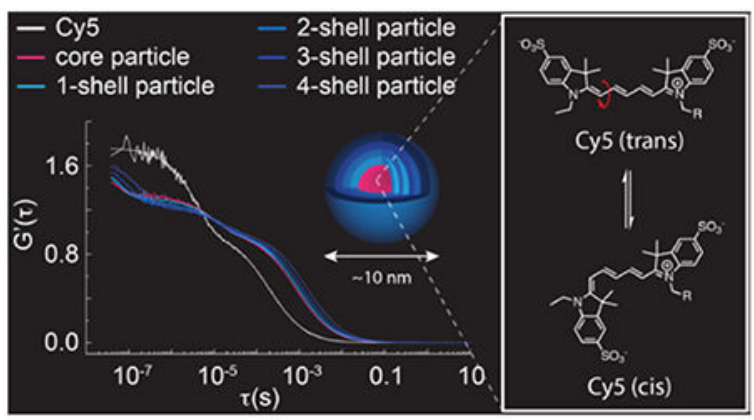

\section{INTRODUCTION}

The ability to control the size of fluorescent organic-inorganic hybrid silica nanoparticles (SNPs) down to a few nanometers, along with well controlled particle surface chemistry, has proven to be a synthetic milestone for the translation of SNPs as diagnostic probes into the clinical setting. ${ }^{1-4}$

With the translation of ultrasmall poly(ethylene glycol)-coated (PEGylated) fluorescent SNPs into the clinic comes further need to understand such nanomaterials in more detail. Sub-10 nm SNPs are often functionalized with expensive fluorophores, targeting moieties, or drugs. ${ }^{5-7}$ While providing pivotal advantages for biomedical applications, this presents challenges for traditional in-depth SNP characterization techniques including dynamic light scattering (DLS), transmission electron microscopy (TEM), or zeta-potential measurements. Studies of size, nanoparticle-morphology, chemical composition the development of novel classes of SNP, or degree of functionalization is often accompanied by low signal-to-noise (e.g. in TEM or zeta-potential measurements), material incompatibility due to fluorescence (i.e. in DLS), the need for large sample amounts (e.g. in solid-state nuclear magnetic resonance (ssNMR) spectroscopy), or cost- and time-intensive experimental procedures (e.g. in cryo-TEM,${ }^{8}$ or synchrotron-based SAXS analysis ${ }^{9}$ ). In addition, the development of novel classes of SNP materials typically requires many iterations of the particle batch synthesis and characterization loop, exacerbating these issues with traditional approaches.

In order to accelerate the development of ultrasmall fluorescent SNPs, fast, inexpensive, sensitive, and versatile characterization techniques are desirable. With the molar mass of such SNPs falling into the regime of macromolecules ( 100k Da), methods typically used for biomolecular research, i.e. chromatography and fluorescence based microscopy and spectroscopy, can present a complimentary path for nanomaterials characterization. ${ }^{10}$ Fluorescence correlation spectroscopy (FCS) stands out among such characterizations techniques for its high information density obtained in a single measurement conducted at nanomolar sample concentrations. FCS accesses information by detecting fluorescence fluctuations around the thermodynamic equilibrium rather than average fluorescence signals. ${ }^{11-14}$ Key to this technique is a very small detection volume, typically a femto to sub-femto liter $\left(10^{-15} \mathrm{~L}\right)$, a thousand times smaller than an eukaryotic cell. Due to the small detection volume and low sample concentrations, no individual molecule or particle is constantly inside the detection volume, allowing to resolve fluorescence fluctuations with high time 
resolution. To interpret fluorescence fluctuations and extract relaxation times of physical processes, the recorded fluorescence traces are tested for self-similarity by an autocorrelation function.

Due to its versatility and minimally invasive nature, FCS has become a well-established technique with a wide spectrum of applications, reaching from the study of photophysical processes, i.e. triplet state dynamics, ${ }^{11}$ photo-induced conformational changes, ${ }^{15}$ to chemical reaction kinetics, ${ }^{16}$ and the study of protein folding, ${ }^{17}$ and living cells. ${ }^{18}$ In principle, every physical process that involves transient non-fluorescent (or weakly fluorescent) states can be studied by FCS. In practice, the accessible relaxation times on a simple confocal setup range from nanoseconds to several hundred milliseconds. Slower processes are typically limited by the emitters' photostability.

The versatility of FCS has led to an increased adoption of FCS in materials science over the last 20 years. Here, FCS is often used employing lag times $>10^{-5} \mathrm{~s}$ for studies of polymer melts, gels and solutions, ${ }^{19,20}$ colloidal and/or photophysical properties of NPs, ${ }^{1,21-24}$ or to study physical adsorption processes to NP surfaces. ${ }^{25,26}$ In contrast, approaches to extend the temporal resolution to fast fluorescence fluctuations (lag times $<10^{-5}$ s) to study NP properties, e.g. rotational diffusion and polarization, remain limited. ${ }^{27,28}$

In this study, we use fast fluorescence fluctuations of the cyanine dye Cy5, caused by photoinduced cis-trans isomerization around its polymethic chain (Figure 1a), to interrogate the local dye environment in ultrasmall organic-inorganic hybrid core-shell SNPs referred to as Cornell dots or simply $\mathrm{C}$ dots with different silica shell architectures. ${ }^{1,21,29,30}$

The photo-physics of cyanine dyes has been studied in detail. ${ }^{31}$ At low excitation intensities $\left(<10 \mathrm{~kW} \mathrm{~cm}^{-2}\right)$, the photophysical relaxation pathways of Cy5 are mostly limited to conformational changes, while the population of triplet states is negligible. ${ }^{32}$ The rates of inter-system crossing and triplet state decay are further reduced upon steric shielding of Cy5 from dissolved oxygen. ${ }^{15}$ In general, large side groups, high viscosity, and low temperatures can retard the conformational reorganizations and reduce the rates for photo-induced isomerization and back-isomerization of cyanine dyes, ${ }^{15}$ making them suitable fluorescence sensors for probing the steric properties of local environments. ${ }^{33,34}$

Here, we synthesized a series of sub-10 nm PEGylated core-shell SNPs in aqueous solution (referred to as C' dots) containing covalently bound Cy5 in the silica core and progressively increasing silica shell thickness before PEGylation. ${ }^{1}$ Using FCS, we show that during the formation process of the silica core a fraction of Cy5 molecules is located near or on the silica core particle surface which in turn is suitable to subsequently probe the deposition of additional silica layers onto the core. In combination with fluorescence lifetime measurements we deduce radiative and non-radiative rates of the Cy5 dye in these particles. We demonstrate that the study of fast dye relaxation kinetics as revealed by FCS is a useful approach to gain detailed insights into the architecture of ultrasmall fluorescent and PEGylated core-shell SNP for applications in biology, nanomedicine, and beyond. 


\section{METHODS}

\section{Chemicals and reagents}

Ammonia solution (2.0 M in ethanol), dimethyl sulfoxide (DMSO), (3-mercaptopropyl) trimethoxysilane (MPTMS), tetramethyl orthosilicate (TMOS), and tetraethyl orthosilicate (TEOS, $299 \%$ ), were purchased from Sigma-Aldrich. Methoxy-terminated poly(ethylene glycol)-silane (mPEG-silane, molar mass of $\sim 0.5 \mathrm{~kg} / \mathrm{mol}$ ) was purchased from Gelest. Cy5meleimide florescent dye and $\mathrm{Cy} 3$-meleimide florescent dye were purchased from GE Healthcare. All chemicals were used as received. Deionized (DI) water $(18.2 \mathrm{M} \Omega \cdot \mathrm{cm})$ was generated using a Millipore Milli-Q system.

\section{Synthesis of fluorescent and PEGylated core-shell silica nanoparticles}

Fluorescent core-shell silica nanoparticles were synthesized according to a modified previously published synthesis protocol. ${ }^{1}$ Core particle synthesis: For the core particle synthesis $0.367 \mu \mathrm{mol} \mathrm{Cy5-maleimide} \mathrm{are} \mathrm{conjugated} \mathrm{to} \mathrm{MPTMS} \mathrm{via} \mathrm{sulfhydryl-maleimide}$ click chemistry under nitrogen atmosphere in the dark overnight (molar ratio dye: MPTMS $=1: 25)$. Then, for a $10 \mathrm{~mL}$ batch synthesis $0.43 \mathrm{mmol}(68 \mu \mathrm{L})$ of TMOS are added to $10 \mathrm{~mL}$ basic DI water ( $\mathrm{pH} 8.5$ ) in a $25 \mathrm{~mL}$ round bottom flask under vigorous stirring, immediately followed by the addition of all previously prepared Cy5-silane. The precursors are left to react at room temperature under vigorous stirring in the dark for 12 hours. The particle dispersion is then transferred to a $100 \mathrm{~mL}$ round bottom flask and diluted with $40 \mathrm{~mL}$ DI water (1:4). Before the growth of a silica shell, an aliquot of $10 \mathrm{~mL}$ is separated for PEGylation ("core particle"). PEGylation: During PEGylation SNPs need to transition from a state of charge stabilization to a state of steric stabilization. In the transition from one state to the other, SNPs are exceptionally vulnerable to aggregation ("valley of death"). ${ }^{25}$ Therefore, it is important that PEGylation kinetics are fast and sufficient PEG molecules are provided to fully cover a SNP. It has been shown that small polyethylene glycol molecules (molar mass of $\sim 500 \mathrm{~g} / \mathrm{mol}$ ) non-covalently adsorb to the surface of SNPs with high adsorption rates by establishing multiple hydrogen bonds between ethoxy units and silica silanol groups in aqueous solutions. ${ }^{25}$ To account for this mechanism, $0.115 \mathrm{mmol}$ of mPEG-silane (MW $500 \mathrm{~g} / \mathrm{mol}$ ) are rapidly added to the $10 \mathrm{~mL}$ aliquot followed by dropwise addition of another $0.115 \mathrm{mmol}$ of $\mathrm{mPEG}$-silane. The purpose of a two-step PEG-addition is to avoid PEG self-aggregation which would reduce the concentration of free PEG monomers in solution. After PEG-addition, the reaction is left stirring at room temperature in the dark for 12 hours. To promote further covalent bond formation between the silane group of mPEG-silane and SNP surface silanol groups, a subsequent 8 hours $80^{\circ} \mathrm{C}$ heating step is followed. ${ }^{25}$ Silica shell growth: The $\mathrm{pH}$ of the $40 \mathrm{~mL}$ left-over particle dispersion is adjusted to $\mathrm{pH} 9$ by an aqueous ammonia solution $(0.02 \mathrm{M})$. To reduce hydrolysis kinetics and increase the control of monomer addition to existing core particles, a TEOS:DMSO dilution (1:10) is dosed into the solution with an auto-dispenser (EDOS 5222, Eppendorf) at a rate of $10 \mu \mathrm{L}$ per $15 \mathrm{~min}$ for 50 times. After the last addition, the reaction is left for another $30 \mathrm{~min}$ before an aliquot of $10 \mathrm{~mL}$ is separated (" 1 shell particle"). The next 3 shells were added in the same way. After each TEOS addition, an aliquot of $10 \mathrm{~mL}$ was taken (with the exception of the last aliquot). The respective rates were: $75 \times(10 \mu \mathrm{L} / 15 \mathrm{~min}$ ) ("2-shell particle"), $70 \mathrm{x}$ (15 $\mu \mathrm{L} / 15 \mathrm{~min})$ ("3-shell particle"), $140 \times$ ( $15 \mu \mathrm{L} / 15 \mathrm{~min})$ ("4-shell particle"). The increased 
TEOS amounts account for the cubic scaling of particle volume with particle radius. All particles were PEGylated according to the core particle protocol (vide supra).

\section{Particle dialysis}

After PEGylation, the particle dispersions were cooled down to room temperature and dialyzed to remove small unreacted precursors. Each sample was transferred to a dialysis membrane tube (Pierce, 10k MWCO) and placed in $2 \mathrm{~L}$ of slowly stirring DI water in the dark for at least 6 hours. Water was exchanged for a minimum of three times before further steps were taken.

\section{Gel permeation chromatography}

Gel permeation chromatography (GPC) served as a preparative and analytical tool to monitor the dispersity of a particle reaction. Based on different elution times of different species in the reaction mixture, the core-shell silica nanoparticles can be separated from unreacted precursors or PEG aggregates (see Results and Discussion). A BioLogic LP system equipped with a $275 \mathrm{~nm}$ UV detector was used. The stationary phase was a Superdex 200 resin (GE Healthcare) in a $2 \mathrm{~cm} \times 20 \mathrm{~cm}$ glass column operated at a flow rate of $2 \mathrm{~mL} /$ $\mathrm{min}$. The mobile phase was a $5 \%(\mathrm{w} / \mathrm{v})$ sodium chloride aqueous solution. Particle concentrations were increased with a spin-filter (GE healthcare Vivaspin, 30k MWCO) before sample loading. Typical injection volumes were 500 to $600 \mu \mathrm{L}$. Fractions at different elution times were collected by a BioFrac fraction collector. For long time storage, all particles were transferred to DI water using a spin-filter by exchanging solvent at least five times.

\section{Steady state absorption and emission spectroscopy}

Absorbance spectra were recorded in DI water on a Varian Cary 5000 spectrophotometer in a $3 \mathrm{~mL}$ quartz cuvette against a reference quartz cuvette with DI water. The absorption of particles and dyes were adjusted to the same optical density before emission spectra were recorded. Optical density values were kept between 0.01 and 0.06 for all samples. Emission spectra of the same samples were recorded on a Photon Technologies International Quantamaster spectrofluorometer. Samples containing Cy5 were excited at $645 \mathrm{~nm}$ and recorded from $655 \mathrm{~nm}$ to $800 \mathrm{~nm}$. All emission spectra were corrected for wavelength dependent detector quantum efficiency differences.

\section{Fluorescence correlation spectroscopy}

FCS setup-A $635 \mathrm{~nm}$ (Cy5) continuous wave solid state laser (LDCU5/8283, Power Technology, Inc.) was reflected by a dichroic mirror and focused onto the object plane of a water immersion microscope objective (Zeiss Plan-Neofluar 63x NA 1.2). The laser power was controlled by inserting neutral OD filters with varying attenuation into the beam path. The laser power was measured by a power meter before the objective. The fluorescence was collected by the same objective and spatially filtered by a $50 \mu \mathrm{m}$ pinhole located at the image plane. The fluorescence was then split into two paths with a beam splitter, spectrally filtered by two separate $665 \mathrm{~nm}$ long pass filters (ET665lp, Chroma), and detected by two avalanche photodiode detectors (SPCM-AQR-14, PerkinElmer). To avoid effects of 
afterpulsing, the two fluorescence time traces were cross-correlated by a hardware correlator card (Flex03LQ, Correlator.com). This allowed for time resolution of $15 \mathrm{~ns}$.

Data fitting-Autocorrelation functions (ACFs) were fitted using a Levenberg-Marquardt non-linear least squares algorithm. ${ }^{35} \mathrm{ACFs}, G^{\prime}(\tau)$, of core-shell SNPs were fitted with a model accounting for translational diffusion, photo-induced cis-trans isomerization, and rotational diffusion:

$$
\begin{aligned}
& G^{\prime}(\tau)=1+ \\
& \frac{1}{N_{m}}\left(\frac{1}{1+\tau / \tau_{D}}\right)\left(\frac{1}{1+\tau /\left(\tau_{D} k^{2}\right.}\right)^{1 / 2} \\
& \left(1+\alpha^{\prime} \exp \left(\tau / \tau_{P}\right)\right) \\
& \left(1+\alpha_{R o t} \exp \left(\tau / \tau_{R o t}\right)\right)
\end{aligned}
$$

$N_{m}$ denotes the average dye or particle number within the observation volume, $\tau_{\mathrm{D}}$ is the characteristic diffusion time through the ellipsoidal observation volume defined by the structure factor $\kappa=\omega_{z} / \omega_{x y}$ with axial $\left(\omega_{z}\right)$ and radial $\left(\omega_{x y}\right)$ radii. The third line of equation 1 describes fast relaxation processes caused by photo-induced cis-trans isomerization. Fluctuations in fluorescence arise from transitioning between a fluorescent transconformation and a non-fluorescent cis-conformation. $a^{\prime}$ is the pre-exponential amplitude of Cy5 cis-trans isomerization. The transition between the two states is characterized by the relaxation time $\tau_{p}$. The last line in equation 1 describes a second fast process assigned to particle rotation that can be expressed in a first approximation as a single exponential. ${ }^{36-39}$ $a_{R o t}$ is the pre-exponential amplitude of particle rotation, and $\tau_{R o t}$ the characteristic rotational diffusion time. This is assuming a linear dipole emission of a spherical particle. ACFs of free dyes were fitted without the contribution of rotational diffusion. ACFs were normalized for the mean number of particles, $N_{m}$, diffusing through the observation volume:

$$
G^{\prime}(\tau)=(G(\tau)-1) \quad N_{m}
$$

Calibration of observation volume-Before each measurement, the effective FCS observation volume, $V_{\text {eff }}$, was calibrated with a standard dye with known diffusion coefficient. A dye stock solution in DMSO was diluted with DI water to nanomolar concentrations. AlexaFluor 647 (diffusion coefficient $3.310^{-6} \mathrm{~cm}^{2} \mathrm{~s}^{-1}$ ) 40 was used as calibration dye for the above mentioned excitation-emission combination at room temperature $\left(20 \pm 1{ }^{\circ} \mathrm{C}\right)$. Typical diffusion times through the observation volume were 200 $\pm 5 \mu$ s (AlexaFluor 647). Calibration and measurements were recorded at low excitation intensities of $5 \mathrm{~kW} \mathrm{~cm}^{-2}$ (Cy5) to avoid singlet-triplet transitions. 
Sample Preparation and measurement-FCS samples were prepared by diluting a stock solution of particles $(\sim 15 \mu \mathrm{M})$ with DI water. Before the FCS measurements an absorption spectrum of the diluted sample was recorded. The same sample was further diluted to nanomolar concentrations. $200 \mu \mathrm{L}$ of this dilution were placed into a $35 \mathrm{~mm}$ glass bottom dish (MatTek Corporation, No. 1.5 coverslip, $10 \mathrm{~mm}$ glass diameter) to be placed onto the objective. Each sample was measured five times in five individual runs, each run was $30 \mathrm{~s}$ long.

Determination of diffusion constant and particle diameter-The typical diffusion time, $\tau_{D}$, as obtained from the fit was used to determine the diffusion constant, $D$, while the Stokes-Einstein relation was applied to determine the particle hydrodynamic diameter, $d$ :

$$
\begin{gathered}
D=\frac{\omega_{x y}{ }^{2}}{4 \tau_{D}} \\
d=2 \frac{k_{B} T}{6 \pi \eta D}
\end{gathered}
$$

where $k_{B}$ stands for the Boltzmann's constant, $T$ is the absolute temperature, and $\eta$ is the dynamic viscosity. A first order approximation of the rotational diffusion constant is: ${ }^{37}$

$$
D_{R o t}=\frac{1}{6 \tau_{R o t}}
$$

where all higher order contributions of the angular momentum $(1>1)$ are ignored. Particle hydrodynamic diameters were determined with the Stokes-Einstein relation for rotational diffusion:

$$
d=2\left(\frac{k_{B} T}{8 \pi \eta D_{\text {Rot }}}\right)^{1 / 3}
$$

Characterization of cis-trans isomerization-Because each dye undergoes independent isomerization, despite possible co-diffusion on one particle, the measured amplitude of isomerization, $a^{\prime}$, is linearly dependent on the average number of dyes per particle, $n_{m} \cdot{ }^{15,41}$ The amplitude of isomerization for a single dye, $a_{P}$, is related by $a^{\prime}=$ $a_{P} / n_{m}$ and the fraction of cyanine dyes being in their cis conformation, $\mathrm{P}$, by $P=a_{P} /(1+$ $a_{P}$ ). To determine $n_{m}$, the measured optical density of each sample was compared to the mean particle concentration as obtained by FCS:

$$
n_{m}=\frac{C_{A b s}}{\langle C\rangle_{F C S}}
$$

with 


$$
\langle C\rangle_{F C S}=\frac{N_{m}}{V_{e f f}}
$$

and

$$
V_{e f f}=\pi^{3 / 2} \omega_{x y}^{2} \omega_{z}
$$

\section{Transmission electron microscopy and image analysis}

Transmission electron microscopy (TEM) images were taken on a FEI Tecnai T12 Spirit electron microscope operated at an acceleration voltage of $120 \mathrm{kV}$. Sample grids were prepared by dropping $10 \mu \mathrm{L}$ of a 50:50 particle and ethanol dispersion onto a TEM grid (EMS, carbon film 300 mesh on copper grids). Sample grids were allowed to dry for at least two hours before they were imaged.

Nanoparticle size distributions were calculated by measuring the area of 200 to 300 nanoparticles per sample, then converting to diameter. Outliers were eliminated if the data point was lower than the $1^{\text {st }}$ quartile - inner quartile range $(\mathrm{IQR}) * 1.5$ or higher than $3^{\text {rd }}$ quartile $+\mathrm{IQR} * 1.5$ then plotted as a $0.1 \mathrm{~nm}$ binned histogram.

\section{Fluorescence lifetime measurements}

Fluorescence lifetimes were taken on an Edinburgh Instruments FLS1000 spectrometer at sample concentrations of $0.5 \mu \mathrm{M}$ to $1 \mu \mathrm{M}$ in deionized water and in a $3 \mathrm{~mL} 10 \mathrm{~mm}$ pathlength quartz cuvette at 90 degrees. Particle samples were excited using a $405 \mathrm{~nm}$ pulsed excitation (Edinburgh Instruments EPL-405 pulsed diode laser with 500 ps pulsewidth, 5 $\mathrm{mW}$ ) operated at a $500 \mathrm{~ns}$ pulse period. Fluorescence was collected with a photon counting photomultiplier tube and decay curves were fitted in the Edinburgh Instruments software using

$$
I(t)=\int_{-\infty}^{t} \operatorname{IRF}\left(t^{\prime}\right) \sum_{i=1}^{n} \alpha_{i} \exp \left(\frac{-t-t^{\prime}}{\tau_{i}}\right) d t
$$

where $\mathrm{I}(\mathrm{t})$ is the fluorescence decay function, $\operatorname{IRF}\left(t^{\prime}\right)$ the instrument response function, $a_{i}$ is the amplitude of the i-th lifetime, $\tau_{i}$, and $n$ is the number of fitting components. The $I R F$ was determined using $40 \mathrm{~nm}$ SNPs. The fit quality was evaluated based on $\chi^{2}$ values. All decay curves were fitted to a bi-exponential decay model $(\mathrm{n}=2)$.

\section{Determination of relative fluorescence quantum yields}

The relative fluorescence quantum yield, $\Phi_{F}$, for SNPs was determined using a comparative method against free Cy5 dye $\left(\Phi_{F}=0.20\right)$ as a standard. ${ }^{42,43}$ 


$$
\Phi_{F, i}=\Phi_{F, r e f} \frac{\mathrm{A}_{r e f}}{\mathrm{~A}_{i}} \int_{\mathrm{I}_{r e f}(\lambda) \mathrm{d} \lambda} \mathrm{I}(\lambda \lambda
$$

where $\int \mathrm{I}_{i}(\lambda) \mathrm{d} \lambda$ is the integrated mean fluorescence intensity from of a sample, $i$, or a reference, ref. Data were recorded in deionized water and at room temperature using absorption values between 0.01 and 0.09 . Samples were excited at $620 \mathrm{~nm}$ and fluorescence recorded and integrated from $630 \mathrm{~nm}$ to $800 \mathrm{~nm}$. Relative fluorescence quantum yields by FCS, $\varphi_{F}$, were determined via the mean photon counts of the APDs normalized for the mean number of dyes or particles, $n_{m}$, in the observation volume.

\section{Determination of rate constants}

Radiative and non-radiative rates were determined from their relations to measured fluorescence quantum yield and fluorescence lifetime:

$$
\begin{gathered}
k_{r}=\frac{\Phi_{F}}{\tau_{F}} \\
k_{n r}=\frac{\left(1-\Phi_{F}\right)}{\tau_{F}}
\end{gathered}
$$

\section{RESULTS AND DISCUSSION}

\section{Particle synthesis and chromatographic work up}

A series of ultrasmall and PEGylated core-shell SNPs (C' dots) with precisely controlled silica shell thickness was first synthesized in aqueous solutions according to a previously reported method. ${ }^{1}$ This method is derived from the principle of living polymerization, that is used for the synthesis of organic polymers with low molar mass/size dispersity: ${ }^{44}$ In order to obtain narrow size distributions, all species have to start to grow at the same time, and have to be terminated at the same time. To that end, first the silane precursor TMOS was added into basic aqueous solution, immediately followed by Cy5-silane addition. The exposure to water causes fast hydrolysis and fast condensation of TMOS leading to rapid seed particle nucleation, followed by particle growth via monomer addition. Then, either further particle growth was suppressed/terminated by the rapid addition of mPEG-silane leading to sterically stabilized PEGylated particles which will be referred to as "core particles" in the following, or further silane precursor was added in small doses to slowly deposit one or more silica shells around the existing particle cores before final PEGylation. Figure $1 \mathrm{~b}$ depicts the principle of the core particle synthesis and shell addition using the silane precursors TMOS and TEOS, respectively. Key for the shell addition is keeping silane precursor concentrations 
below the threshold for secondary particle nucleation at any time. TEOS in comparison to TMOS hydrolyzes slower. This allows the precursor to spread throughout the solution before hydrolysis, keeping the local concentrations low enough to suppress secondary particle formation, and leading to steady primary particle growth upon TEOS dose addition. After the desired amount of TEOS was added, an aliquot of the particle solution was removed and sterically stabilized by mPEG-silane addition. In this way five different particles with increasing shell thicknesses were synthesized originating from the same core synthesis (See Materials and Methods): the core-particles and particles with one, two, three, or four additional silica shells, respectively (Figure 1b).

Insufficient charge stabilization during synthesis and non-optimized PEGylation protocols can lead to a highly uncontrolled particle dispersity. ${ }^{25}$ Gel permeation chromatography (GPC) is an excellent analytical tool to study particle size distributions and heterogeneities, as well as a preparative tool to separate any unreacted precursors, fluorophores, aggregates, secondary particles, or mPEG-silane from the native solution. ${ }^{1}$ GPC separates analytes based on hydrodynamic radii, by eluting analytes together with a mobile phase through a porous stationary phase. Smaller analytes remain longer in the stationary phase and elute at later times. To clean the different core-shell SNPs from all possible side-products, we submitted every sample to two GPC runs. The GPC elugram of the first run (Figure 1c) consisted of mainly three peaks for every sample, consistent with earlier observations. ${ }^{1}$ The first peak at around 9 min was a sharp peak that did not change for different samples, and likely stems from PEG aggregates and/or undyed particle aggregates (Figure S1, Supporting Information). Across the particle series, the second peak displayed a clear shift towards shorter elution times with additional silica shells (from $15 \mathrm{~min}$ to $12 \mathrm{~min}$ ). It is therefore assigned to the main core-shell SNP peak. Some of the core-shell SNP peaks, i.e. for the 2shell and 3-shell particles, showed a minor right shoulder. We suspect this shoulder to be a result of particle PEGylation of aliquots before GPC (e.g. mPEG-silane adducts), since the 4-shell particle does not display any such shoulder. It is likely due to an excess of unreacted precursor that was still present in the reaction at the time of PEGylation. Finally, the third peak was a broad low-intensity band positioned between $17 \mathrm{~min}$ and $25 \mathrm{~min}$ (indicated by a black arrow) and was present in all samples. This band is originating from monomeric and low molar mass precursors (Figure S1, Supporting Information). ${ }^{1}$

Shifts of the main GPC particle peak towards shorter elution times were expected due to particle growth through silica shell additions. In order to eliminate the shoulder in the particle size distributions and optimize sample quality, a two-sigma fraction from each main particle peak was collected (grey area under curves in Figure 1c) and submitted to another GPC run (Figure 1d). These elugrams now all showed symmetric shapes. As a cross-check, from these second GPC runs further two-sigma fractions were taken and again submitted to subsequent GPC runs. Within the variance of these measurements, the main particle peak positions remained unchanged, indicating successful steric stabilization of the various coreshell SNPs via the PEGylation process. ${ }^{25}$

\section{Particle characterization with afterpulse-corrected fluorescence correlation}

spectroscopy-After sample preparation, fluorescence correlation spectroscopy (FCS) was used to characterize the five core-shell SNPs in detail. FCS derives information on 
dynamic processes from spontaneous fluctuations of fluorescence over time. ${ }^{12-14}$ The observation of fluctuations in intensity is realized by restricting the detection of fluorescence to a very small observation volume. Figure 2a illustrates the effective observation volume, $V_{\text {eff }}$, that is created by focusing a laser beam with a confocal lens. $V_{\text {eff }}$ is defined as $V_{\text {eff }}=$ $\pi^{3 / 2} \omega_{x y}^{2} \omega_{z}$, with $\omega_{x y}$ and $\omega_{z}$ being the radial and axial $1 / \mathrm{e}^{2}$ radii from the center of a Gaussian-shaped focused laser beam. Only when a particle diffuses through $V_{\text {eff }}$ fluorescence is emitted. This yields high signal-to-noise fluorescence signals and allows detection of fluctuations from single particles. The information about physical processes is subsequently extracted from the self-similarity of the fluorescence time trace after a lag time, $\tau$, by analyzing the fluctuations with the normalized autocorrelation function, $G(\tau)$ :

$$
\begin{aligned}
& G(\tau)=\frac{\langle I(t) I(t+\tau)\rangle}{\langle I(t)\rangle^{2}}=\frac{\langle[\langle I(t)\rangle+\delta I(t)][\langle I(t)\rangle+\delta I(t+\tau)]\rangle}{\langle I(t)\rangle^{2}}=1 \\
& +\frac{\langle\delta I(t) \delta I(t+\tau)\rangle}{\langle I(t)\rangle^{2}}
\end{aligned}
$$

Here $I(t)$ is the detected fluorescence intensity at time $t,\langle I(t)\rangle$ is the mean fluorescence intensity over time, and $\delta I(t)$ is the fluctuation of $I(t)$ around its mean.

Figure $2 \mathrm{~b}$ shows a typical confocal FCS setup. A continuous wave laser is reflected by a dichroic mirror (DM) and then focused by the microscope objective (OB) down to the diffraction limit. The same objective that previously focused the laser, now collects the fluorescence. The Stokes-shifted fluorescence passes the same DM, is then spatially filtered from scattered photons by a pinhole $(\mathrm{PH})$ in the image plane, and spectrally filtered from excitation photons by an emission long pass filter (LPF). A fluorescence time trace is detected by an avalanche photodiode detector (APD), and auto-correlated by a correlator card (Figure 2c). Figure 2d shows the respective autocorrelation function/curve (ACF).

When a photon reaches the sensitive APD, a photo current is created, that often cause a second follow-up electrical pulse (afterpulsing). These effects occur with a periodicity of 10 ns to $100 \mathrm{~ns}$, and in this time window lead to a large background in the ACF (curve (i) in Figure $2 \mathrm{~d}$ ). To accurately measure fast transient states $(\tau<0.5 \mu \mathrm{s})$, effects by detector afterpulsing therefore need to be avoided. This can be achieved by splitting the fluorescence signal into two paths and detecting each path with a separate detector. ${ }^{45}$ The two fluorescence time traces (Figure 2c) are then cross-correlated, uncovering the underlying photophysical phenomena in the ACF (curve (ii) in Figure 2d). For a nanoparticle that is diffusing in three dimensions through a sample observation volume, and carrying a fluorescent dye molecule with the ability to undergo photo-induced conformational changes, the contributions in the ACF can be roughly divided into three regions on the lag time axis (Figure 2d): Region I is reflecting the translational diffusion of particles ( $\tau>10 \mu \mathrm{s})$, region II corresponds to photo-isomerization processes $(10 \mu \mathrm{s}>\tau>0.1 \mu \mathrm{s})$, and region III detects effects from particle rotation $(\tau<0.1 \mu \mathrm{s}$ ). The normalized diffusion autocorrelation function can then be described as:

$$
G(\tau)=G(0) G_{D}(\tau) G_{P}(\tau) G_{R o t}(\tau)+1
$$


with

$$
\begin{gathered}
G(0)=N_{m}^{-1}=V_{e f f}{ }^{-1}\langle C\rangle^{-1} \\
G_{D}(\tau)=\left(1+\tau / \tau_{D}\right)^{-1}\left(1+\tau /\left(\tau_{D} \kappa^{2}\right)\right)^{-1 / 2} \\
G_{P}(\tau)=\left(1+\alpha^{\prime} \exp \left(\tau / \tau_{P}\right)\right) \\
G_{R o t}(\tau)=\left(1+\alpha_{R o t} \exp \left(\tau / \tau_{R o t}\right)\right)
\end{gathered}
$$

All where $N_{m}$ is the mean number of emitters in the effective observation volume, $V_{\text {eff }}$, at the mean sample concentration $\langle C\rangle, \tau_{D}$ is the average particle diffusion time through the observation volume, $\kappa=\omega_{z} / \omega_{x y}$ is the structure factor defined by the observation volume dimensions, $a^{\prime}$ is pre-exponential amplitude associated with cis-trans photoisomerization, $\tau_{P}$ is the relaxation time of a single cyanine dye molecule undergoing photo-isomerization, $a_{R o t}$ is the pre-exponential amplitude of particle rotation, and $\tau_{R o t}$ is the characteristic particle rotation time.

In the following, for our set of ultrasmall fluorescent and PEGylated core-shell SNPs FCS was used to characterize particle hydrodynamic diameter, $d_{F C S}$, particle concentrations, cistrans isomerization, and particle rotation. Figure 3 summarizes the information obtained from the FCS measurements on these SNPs with embedded Cy5 molecules. Figure 3a compares the ACFs for free Cy5 dye, the core particle, and the four core-shell particles, with corresponding fits according to equation 15 superimposed over the data. The relative shift of the SNPs to longer lag times relative to free Cy5 indicates increasingly slower diffusion (enlarged in inset (i) of Figure 3a). The average translational diffusion constants were determined from the characteristic diffusion times and translated into particle hydrodynamic diameters using the Stokes-Einstein relation. Average hydrodynamic diameters ranged from $5.0 \pm 0.1 \mathrm{~nm}$ (core particle), $5.6 \pm 0.1 \mathrm{~nm}$ (1-shell particle), $6.3 \pm 0.1 \mathrm{~nm}$ (2-shell particle), $7.4 \pm 0.1 \mathrm{~nm}$ ( 3 -shell particle), to $9.5 \pm 0.2 \mathrm{~nm}$ (4-shell particle) (Figure $3 \mathrm{~b}$ ). The average shell thicknesses for each core-shell particle were calculated from the incremental particle growth. These were $0.3 \mathrm{~nm}, 0.7 \mathrm{~nm}, 1.2 \mathrm{~nm}$, and $2.3 \mathrm{~nm}$ for the 1-, 2-, 3-, and 4-shell coreshell SNPs, respectively, suggesting a synthesis precision down to the deposition of a single atomic $\mathrm{SiO}_{2}$ layer between particles $(\sim 0.4 \mathrm{~nm}){ }^{1}$

For comparison, particle diameters, of core, 2-shell, and 4-shell particles were determined using transmission electron microscopy (TEM) as $3.4 \pm 0.4 \mathrm{~nm}, 3.9 \pm 0.5 \mathrm{~nm}$, and $8.0 \pm 1.1$ $\mathrm{nm}$, respectively (Figure $\mathrm{S} 2$ ). These TEM based diameters, $d_{T E M}$, are expected to be smaller than those determined by FCS as they only account for the inner silica part of the particles, but not for the PEG layer nor for the water shell dragged with the particles and detected in hydrodynamic diameter measurement techniques such as FCS. As the particle diameters increase the ratio between particle-surface and particle-volume decrease. Consequently, the 
contributions from surface PEGylation and solvation layer to $d_{F C S}$ decrease and $d_{F C S}$ and $d_{T E M}$ converge.

At fluorescence fluctuations below $\tau<100 \mathrm{~ns}$ the embedded Cy5 dye cannot be assumed to be a rotationally isotropic emitter anymore, since the change in the orientation of the Cy5 dipole moment is linked to the rotation of the entire particle, whose mobility is significantly slowed down compared to a free Cy5 dye. The afterpulse-corrected ACF (region (iii) in Figure 3a) reveals the time scale and population of rotational diffusion of the entire particles. With increasing particle diameter the relaxtion time of this rotational diffusion process ( $\tau R o t$ ) is noticeably shifting towards larger lag times and manifests itself by slightly increasing amplitude, $a$ Rot (Table S1). The molecular rotation motion of free Cy5 dye lies beyond the accessible time resolution of our setup and therefore does not display any contribution to the measured ACFs.

We also used $\tau_{R o t}$ to determine the particle sizes and shell thicknesses with the assumption of a linear dipole emission and a negligible contribution of higher order angular momentum 1 $(1>1) .{ }^{36-39}$ Figure $3 b$ illustrates a comparative graph of particle diameters plotted against shell thickness, as obtained from translational and rotational diffusion, respectively. Three observations were made: First, the average particle diameters as calculated from particle rotation are systematically larger than those from translational diffusion. Second, the errors associated with the diameters determined from rotational diffusion decrease with increasing particle sizes. Lastly, the determined average shell thickness almost perfectly matches the ones determined by translational diffusion. The larger errors for smaller particles are expected due to fitting of only the onsets of the rotational diffusion relaxation (see Figure 3a). The systematic deviations of particle diameters as determined by translational versus rotational diffusion can be explained by the presence of more than one emitter per particle. The transition dipole moments of multiple Cy5 dye molecules in one particle are likely not aligned, leading to enhanced fluorescence signal despite a rotating particle. This additional fluorescence signal is reflected in an apparently larger particle diameter than determined by translational diffusion.

Unlike free Cy5 dye, where dye molecules are expected to diffuse independently of each other, SNPs can carry multiple Cy5 molecules. The average number of Cy5 dyes per SNP $\left(n_{m}\right)$ can be calculated by dividing the concentration of fluorophores as obtained from steady-state absorption spectroscopy measurements by the mean concentration of SNPs as determined by FCS measurements for the same sample. This analysis revealed about 1.7 \pm 0.1 dyes per SNP for all particles (Figure 3c).

The rotational diffusion amplitude, $a_{R o t}$, should be independent from the number of dyes per particle. However, we find that the amplitude slightly increases with progressive silica deposition. Yin et al. found a dye number dependence of rotational diffusion amplitudes for a TMR labeled DNA Holliday junction, and concluded that the amplitude of rotational relaxation is dye number dependent only if vibrational motion and wobbling are faster or on the order of rotational motion. ${ }^{41}$ With this assumption and the same number of dyes for all core-shell SNPs, we infer that the increasing amplitude, $a_{R o t}$, suggests a correlation between the shell thickness and a decrease in dye mobility within the particles, both by the 
suppression of isomerization, as well as by the restriction of vibrational movement and wobbling.

The brightness of the core particle is a factor 3 higher than that of the free dye and asymptotically increases to nearly a factor of 4 for the largest silica shell thickness (Figure 3d). By calculating the brightness per dye, one can see that the average dye brightness increases with increasing shell thickness (Figure 3e). This behavior asymptotically approaches an upper threshold at a factor of around 2.6 times the brightness of free $\mathrm{Cy} 5$, or in other words, the deposition of more silica beyond this upper threshold is not expected to influence the brightness of Cy5 much further (Figure 3f). The increase of fluorescence brightness (brightness enhancement) of organic dyes upon covalent encapsulation in silica has been described before and is dependent on the silica architecture and the chemical structure of the dye. ${ }^{1,21,46,47}$ From the fact that the brightness per dye changes upon further silica deposition we conclude that some Cy5 molecules are originally located on the surface of the core particle or are only partially embedded within the silica network of the core particle. Two arguments support this hypothesis. First, during the synthesis (pH 8.5), SNPs are stabilized through Coulomb repulsion of negative surface charges while Cy5 dye itself also has a net charge of minus one. It is expected that the associated charge repulsion hinders the full covalent dye incorporation of Cy5-silane into the particle core. This is supported by the limited dye incorporation of Cy5 found by GPC analysis of the synthesis solution shown in Figure S1b. Second, the core particle has a radius of only about $2.5 \mathrm{~nm}$, while the measured size of Cy5 along the long molecular axis is about $1.2 \mathrm{~nm}$. With an average number of approximately two Cy5 molecules per particle it appears that the likelihood is low of all fluorophores being fully surrounded by silica.

This conclusion of near surface dye molecules was further supported by behavior of the FCS curves in region II of Figure 3a (see also inset (ii) in this figure). The fluorescence fluctuations in that region are due to photo-induced conformational changes of the Cy5 dye. Since multiple embedded fluorophores can co-diffuse with one particle but undergo isomerization independently from each other, the measured amplitude of isomerization, $a^{\prime}$, scales with $1 / n_{m}$. By determining the number of dyes per particle $\left(n_{m}\right)$ one can calculate the fraction of Cy5 dyes being in their cis state, $P$ (see methods). ${ }^{41}$ With increasing shell thickness, we can observe a clear reduction in the second amplitude of the ACF (see arrow in inset (ii) of Figure $3 \mathrm{a}$ ). Figure $3 \mathrm{~g}$ shows the comparative results of the cis-conformers fraction, $P$. Starting from nearly $50 \%$ for $\mathrm{Cy} 5$ dye in solution, the fraction $\mathrm{P}$ asymptotically decreased to reach a value of about $27 \%$ for the 4 -shell particle. This behavior can explain the previously described changes in fluorescence brightness. Cis-conformers are only weakly fluorescent and therefore don't contribute significantly to the overall particle brightness. ${ }^{15}$ With increasing silica shell thickness the likelihood of a rigid silica dye environment increases, prohibiting the spatially demanding conformational changes of Cy5. Interestingly, despite being encapsulated in a particle with a thick shell $(2.3 \mathrm{~nm})$ and seemingly reaching a limit of brightness increase, the fraction of cis conformer, $\mathrm{P}$, is still at $27 \%$. This finding suggests that some Cy5 molecules can still undergo isomerization despite being fully encapsulated. Silica is an amorphous and microporous material when synthesized in water. ${ }^{48}$ It therefore does not surprise that there remain large enough cavities for some dyes to undergo conformational changes. In addition, it is likely that the slow 
deposition of further silica onto existing SNPs (as compared to the faster core reaction) is disturbed by the presence of near-surface dyes. These molecules therefore likely maintain a larger cavity in the silica shell network than dyes encapsulated in the silica core network.

\section{Determination of kinetic rates of dye induced optical phenomena-At the} excitation intensity employed $\left(5 \mathrm{~kW} / \mathrm{cm}^{2}\right)$, the quantum yield of triplet formation is assumed to be low and triplet kinetics are further reduced due to steric shielding of Cy5 from dissolved oxygen by the silica network. Consequently, the energy relaxation pathway in region II can solely be attributed to photo-induced cis-trans isomerization. ${ }^{15}$ Transitions between singlet ground, $\mathrm{S}_{0}$, and singlet excited, $\mathrm{S}_{1}$, states of both dye conformations take place on the nanosecond time scale and cannot be resolved by our FCS setup (temporal resolution $15 \mathrm{~ns})$. The corresponding rates of these processes can be expected to be much faster than the rates of the transition process between the fluorescent trans-state and the nonfluorescent cis-state. Therefore, there is no need for differentiation of singlet state transitions in a kinetic model. The transition between the different conformations can be described in terms of a simple two-state model, Trans $\rightleftharpoons$ Cis, with an effective rate for isomerization, $k_{i s o}$, and an effective rate for back-isomerization, $k_{\text {biso }}$ (Figure 1a). With the determined fractions, $P$, and the relaxation times, $\tau_{P}$, from FCS one can calculate the effective isomerization rates and the equilibrium constant, $\mathrm{K}$ :

$$
\begin{gathered}
\tau_{P}=\frac{1}{k_{i s o}+k_{\text {biso }}} \\
\mathrm{P}=\frac{k_{\text {iso }}}{k_{\text {iso }}+k_{\text {biso }}}=\frac{1}{1+K}
\end{gathered}
$$

All results from this analysis are summarized in Table 1. Both, the rate of isomerization and the rate of back-isomerization are retarded by a factor of 2 to 3 upon Cy 5 encapsulation into the core particle and further reduced with silica shell deposition. In comparison, $k_{i s o}$ is slightly smaller than $k_{\text {biso }}$ and reduces slightly more with dye encapsulation. The equilibrium of photo-induced cis-trans isomerization shifts towards the trans conformation with progressive silica deposition.

In previous studies the increased fluorescence brightness of dye encapsulating SNPs has been explained by an increase in radiative rate, $k_{r}$, and/or a decrease in non-radiative rate, $k_{n I}$, of embedded tetramethyl-rhodamine dye molecules. ${ }^{46}$ Combinations of fluorescence quantum yield, $\varphi_{F}$, and fluorescence lifetime, $\tau_{F}$, were used to determine these rates based on: $k_{r}=\varphi_{F} \tau_{F}$ and $k_{n r}=\left(1-\varphi_{F}\right) / \tau_{F}$. The fluorescence quantum yield was estimated from the brightness enhancement per dye that was obtained by FCS measurements. Since excitation intensities are low, and saturation effects can be expected to be low as well, this should be a reasonable estimation.

Figure S3a compares time resolved fluorescence decay curves for the five different SNPs of this study. All curves were fitted with a bi-exponential decay model (see Table S2). The corresponding fluorescence lifetime values are plotted against the shell thickness in Figure 
4a. The fluorescence lifetime increases with silica shell thickness. The increase is most pronounced between free dye and core particle, but continues with silica shell thickness.

Table 2 summarizes the corresponding radiative and non-radiative rates as obtained from FCS and time resolved fluorescence decay measurements. Most noticeable is a strong decrease of non-radiative rates that correlates with increasing shell thickness. The largest decrease (about a factor of 2.5) is observed upon Cy5 encapsulation into the core particle. However, upon further silica addition, these values decreased by another factor of $\sim 1.5$, reflecting the restricted conformational mobility of $\mathrm{Cy} 5$ dye and supporting the previous hypothesized near-surface location of Cy5 dye molecules in the core particles.

In contrast, radiative rates do not significantly increase. This behavior is very different compared to rhodamine dyes. For example, a 2.3 -fold increase of radiative rates was measured for different silica nanoparticle architectures embedding TRITC dye, ${ }^{46}$ and a 2.1fold increase for calcium phosphate nanoparticles embedding Cy 3 dye. ${ }^{49}$ The values obtained in our study ( 1.0 to 1.2-fold increase) lie far below these literature values. Radiative rate, $k_{I}$, can be estimated by applying an expression derived by Strickler and Berg, ${ }^{50}$ which describes the relationship between $k_{l}$, the excitation spectrum, the emission spectrum, and the square of the refractive index of the surrounding medium (equation 22 in ref. 50). Here, we compared free Cy5 dye in water, $\mathrm{n}_{1}=1.33$, and Cy5 dye encapsulated in silica, $\mathrm{n}_{2}=1.46$. Our calculated values of normalized values for $k_{r}$ were $\sim 1.20$ for all particles, and agreed best with our experimentally determined value for the 4-shell particle, and slightly overestimated the values for the other particles (see Table 2). Since excitation and emission profiles do not significantly change upon silica encapsulation, the difference of radiative rates is mostly due to changes of refractive index. ${ }^{51}$ As mention above, silica is a porous material that can be penetrated by water, especially when the silica thickness is on the order of a few atomic layers. Therefore, the effective refractive index for the core-shell particles probably lies between 1.33 and 1.46, and approaches 1.46 with increasing shell thickness. ${ }^{52}$

For comparison, we determined the same radiative rates and non-radiative rates using steady-state absorption and emission spectroscopy. Figure $4 \mathrm{~b}$ shows the intensity matched steady-state absorption and corresponding emission spectra of Cy5 and Cy5 particles in water. Upon embedding Cy5 into the silica matrix a minor solvatochromic red-shift ( $\sim \mathrm{nm})$ relative to Cy5 in water was observed. This was the case for all particles and did not noticeably change with thicker shell. The emission spectra showed a relative brightness enhancement in comparison to free Cy5 dye, that was more pronounced with increasing shell thickness. The relative fluorescent quantum yields, $\Phi_{F}$, for the SNP were deduced by using the comparative method by Williams et al., ${ }^{42}$ which is based on the integration of corresponding emission spectra for different sample absorbances (Figure S3b). In this case, Cy5 was used as the standard with $\Phi_{F}=0.20$. All results obtained via steady state spectroscopy are summarized in Table 3. We find that FCS and steady-state spectroscopy yield similar results. We conclude that the observed brightness enhancement for the coreshell SNP series is mostly the result of a decrease of non-radiative rates, and only weakly dependent on a minor increase of radiative rates. The relative decrease of non-radiative rates is strongly coupled to the relative decrease of the effective isomerization rates, and therefore 
to the increased rigidity of the silica dye environment. This in turn is consistent with earlier findings for TRITC dye encapsulation in silica. ${ }^{46}$ It is likely that for Cy 5 the reduced capability for conformational changes within the silica matrix is the biggest contributor to the reduction of non-radiative relaxation pathways at the given excitation intensity.

\section{CONCLUSIONS}

In this study, we synthesized and characterized a series of ultrasmall and PEGylated fluorescent core-shell silica nanoparticles (SNPs, C' dots) with covalently encapsulated cyanine Cy5 dye and increasing silica shell thickness using a combination of gel permeation chromatography, GPC, fluorescence correlation spectroscopy, FCS, time-resolved fluorescence decay measurements, and steady state absorption and emission spectroscopy. We learned that a significant fraction of Cy5 dye molecules are located near the surface or on the surface of the silica core used for the synthesis of all these particles.

The photo-induced cis-trans isomerization of Cy5 as monitored by FCS at lag times $<10^{-5} \mathrm{~s}$ allowed to probe the effects of silica deposition at the single atomic layer level onto the core SNP. A silica shell of $2.3 \mathrm{~nm}$, about double the length of Cy5, is sufficient to cover these dyed particles with silica. This leads to a maximum brightness enhancement of 2.6 as compared to free $\mathrm{Cy} 5$ dye in aqueous solution. In these maximum brightness particles still about $27 \%$ of the Cy5 population are able to undergo photo-isomerization, likely in free volume cavities within the NP silica matrix, giving room for further improvements in brightness utilizing alternative synthesis methods.

Results demonstrated that FCS is a powerful tool for the characterization of fluorescent SNPs. Fast measurements, low sample concentrations, relatively low cost and high information density, make FCS compatible with high throughput synthetic approaches for development of ultra-small nanoparticles, i.e. many batch iterations, or instrumental coupling with microfluidic devices for nanoparticle syntheses. In combination with other sensitive techniques, such as high resolution chromatography and single particle methods, new insights into the structure and optical properties of ultra-small SNP can be achieved.

\section{Supplementary Material}

Refer to Web version on PubMed Central for supplementary material.

\section{ACKNOWLEDGEMENTS}

This study was funded by the U.S. Department of Energy, Office of Science, Basic Energy Sciences under Award No. DE-SC0010560. J.A.H. thanks the National Institutes of Health (NIH) for funding under Award No. U54CA199081. This work made use of the Cornell Center for Materials Research shared facilities which are supported through the NSF MRSEC program (DMR-1719875) at Cornell. The authors gratefully acknowledge Dr. W. Zipfel and Dr. L. Estroff (Cornell University) for helpful discussions and kind experimental assistance, as well as Dr. Nirmalya Bag for insightful discussions.

\section{REFERENCES}

1. Ma K; Mendoza C; Hanson M; Werner-Zwanziger U; Zwanziger J; Wiesner U Control of Ultrasmall Sub-10 nm Ligand-Functionalized Fluorescent Core-Shell Silica Nanoparticle Growth in Water. Chem Mater 2015, 27, 4119-4133. 
2. Phillips E; Penate-Medina O; Zanzonico PB; Carvajal RD; Mohan P; Ye Y; Humm J; Gonen M; Kalaigian H; Schoder H et al. Clinical Translation of an Ultrasmall Inorganic Optical-PET Imaging Nanoparticle Probe. Sci Transl Med. 2014, 6, 260 ra149.

3. Choi J; Burns AA; Williams RM; Zhou Z; Flesken-Nikitin A; Zipfel WR; Wiesner U; Nikitin AY Core-Shell Silica Nanoparticles as Fluorescent Labels for Nanomedicine. J Biomed Optics 2007, 12, 064007-1-064007-11.

4. Hartshorn CM; Bradbury M; Lanza G; Nel A; Rao J; Wang AZ; Wiesner U; Yang L; Grodzinski P Nanotechnology Strategies to Advance Outcomes in Clinical Cancer Care. ACS Nano 2018, 12, 24 43. [PubMed: 29257865]

5. Chen F; Ma K; Madajewski B; Zhuang L; Zhang L; Rickert K; Marelli M; Yoo B; Turker M; Overholtzer $\mathrm{M}$ et al. Ultrasmall Targeted Nanoparticles with Engineered Antibody Fragments for Imaging Detection of HER2-Overexpressing Breast Cancer. Nat Commun 2018, 9, 4141. [PubMed: 30297810]

6. Chen F; Zhang X; Ma K; Madajewski B; Benezra M; Zhang L; Phillips E; Turker MZ; Gallazzi F; Penate-Medina $\mathrm{O}$ et al. Melanocortin-1 Receptor-Targeting Ultrasmall Silica Nanoparticles for Dual-Modality Human Melanoma Imaging. ACS Appl Mater Interfaces 2018, 10, 4379-4393. [PubMed: 29058865]

7. Yoo B; Ma K; Zhang L; Burns A; Sequeira S; Mellinghoff I; Brennan C; Wiesner U; Bradbury MS Ultrasmall Dual-Modality Silica Nanoparticle Drug Conjugates: Design, Synthesis, and Characterization. Bioorg Medic Chem 2015, 23, 7119-7130.

8. Ma K; Gong Y; Aubert T; Turker MZ; Kao T; Doerschuk PC; Wiesner U Self-Assembly of Highly Symmetrical, Ultrasmall Inorganic Cages Directed by Surfactant Micelles. Nature 2018, 558, 577580. [PubMed: 29925942]

9. Barteau K; Ma K; Kohle FFE; Gardinier T; Beaucage P; Gillilan R; Wiesner U Quantitative Measure of the Size Dispersity in Ultrasmall Fluorescent Organic-Inorganic Hybrid Core-Shell Silica Nanoparticles by Small-Angle X-ray Scattering. Chem Mater 2018, 31, 643-657.

10. Gardinier TC; Kohle FFE; Peerless JS; Ma K; Turker MZ; Hinckley JA; Yingling YG; Wiesner U High Performance Chromatographic Characterization of Surface Chemical Heterogeneities of Fluorescent Organic-Inorganic Hybrid Core-Shell Silica Nanoparticles. ACS Nano 2018, in press, 10.1021/acsnano.8b07876.

11. Widengren J; Mets U; Rigler R Fluorescence Correlation Spectroscopy of Triplet States in Solution: A Theoretical and Experimental Study. J Phys Chem 1995, 99, 13368-13379.

12. Elson EL; Magde D Fluorescence Correlation Spectroscopy. I. Conceptual Basis and Theory. Biopolymers 1974, 13, 1-27.

13. Magde D; Elson EL; Webb WW Fluorescence Correlation Spectroscopy. II. An Experimental Realization. Biopolymers 1974, 13, 29-61. [PubMed: 4818131]

14. Magde D; Elson E; Webb WW Thermodynamic Fluctuations in a Reacting System - Measurement by Fluorescence Correlation Spectroscopy. Phys Rev Lett 1972, 29, 705-708.

15. Widengren J; Schwille P Characterization of Photoinduced Isomerization and Back-Isomerization of the Cyanine Dye Cy5 by Fluorescence Correlation Spectroscopy. J Phys Chem A 2000, 104, 6416-6428.

16. Hom EFY; Verkman AS Analysis of Coupled Bimolecular Reaction Kinetics and Diffusion by Two-Color Fluorescence Correlation Spectroscopy: Enhanced Resolution of Kinetics by Resonance Energy Transfer. Biophys J 2002, 83, 533-546. [PubMed: 12080140]

17. Michalet X; Weiss S; Jäger M Single-Molecule Fluorescence Studies of Protein Folding and Conformational Dynamics. Chem Rev 2006, 106, 1785-1813. [PubMed: 16683755]

18. Schwille P Fluorescence Correlation Spectroscopy and Its Potential for Intracellular Applications. Cell Biochem Biophys 2001, 34, 383-408. [PubMed: 11898862]

19. Wöll D Fluorescence Correlation Spectroscopy in Polymer Science. RSC Adv 2014, 4, 2447-2465.

20. Nath P; Mangal R; Kohle F; Choudhury S; Narayanan S; Wiesner U; Archer LA Dynamics of Nanoparticles in Entangled Polymer Solutions. Langmuir 2017, 34, 241-249. [PubMed: 29192503]

21. Ow H; Larson DR; Srivastava M; Baird BA; Webb WW; Wiesner U Bright and Stable Core-Shell Fluorescent Silica Nanoparticles. Nano Lett 2005, 5, 113-117. [PubMed: 15792423] 
22. Doose S; Tsay JM; Pinaud F; Weiss S Comparison of Photophysical and Colloidal Properties of Biocompatible Semiconductor Nanocrystals Using Fluorescence Correlation Spectroscopy. Anal Chem 2005, 77, 2235-2242. [PubMed: 15801758]

23. Zhang B; Lan T; Huang X; Dong C; Ren J Sensitive Single Particle Method for Characterizing Rapid Rotational and Translational Diffusion and Aspect Ratio of Anisotropic Nanoparticles and Its Application in Immunoassays. Anal Chem 2013, 85, 9433-9438. [PubMed: 24059451]

24. Kuyper CL; Fujimoto BS; Zhao Y; Schiro PG; Chiu DT Accurate Sizing of Nanoparticles Using Confocal Correlation Spectroscopy. J Phys Chem B 2006, 110, 24433-24441. [PubMed: 17134198]

25. Ma K; Zhang D; Cong Y; Wiesner U Elucidating the Mechanism of Silica Nanoparticle PEGylation Processes Using Fluorescence Correlation Spectroscopies. Chem Mater 2016, 28, 1537-1545.

26. Röcker C; Pötzl M; Zhang F; Parak WJ; Nienhaus GU A Quantitative Fluorescence Study of Protein Monolayer Formation on Colloidal Nanoparticles. Nat Nanotechnol 2009, 4, 577-580. [PubMed: 19734930]

27. Tsay JM; Doose S; Weiss S Rotational and Translational Diffusion of Peptide-Coated $\mathrm{CdSe} / \mathrm{CdS} / \mathrm{ZnS}$ Nanorods Studied by Fluorescence Correlation Spectroscopy. J Am Chem Soc 2006, 128, 1639-1647. [PubMed: 16448137]

28. Fortunati I; Weber V; Giorgetti E; Ferrante C Two-Photon Fluorescence Correlation Spectroscopy of Gold Nanoparticles under Stationary and Flow Conditions. J Phys Chem C 2014, 118, 2408124090.

29. Herz E; Ow H; Bonner D; Burns A; Wiesner U Dye Structure - Optical Property Correlations in Near-Infrared Fluorescent Core-Shell Silica Nanoparticles. J Mater Chem 2009, 19, 6341-6347.

30. Herz E; Burns A; Bonner D; Wiesner U Large Stokes-Shift Fluorescent Silica Nanoparticles with Enhanced Emission over Free Dye for Single Excitation Multiplexing. Macromol Rap Commun 2009, 30, 1907-1910.

31. Levitus M; Ranjit S Cyanine Dyes in Biophysical Research: The Photophysics of Polymethine Fluorescent Dyes in Biomolecular Environments. Q Rev Biophys 2010, 44, 123-151. [PubMed: 21108866]

32. Kohle FFE; Hinckley JA; Li S; Dhawan N; Katt WP; Erstling JA; Werner-Zwanziger U; Zwanziger J; Cerione RA; Wiesner UB Amorphous Quantum Nanomaterials. Adv Mater. 2018, 31, 1806993.

33. Chmyrov V; Spielmann T; Hevekerl H; Widengren J Trans-Cis Isomerization of Lipophilic Dyes Probing Membrane Microviscosity in Biological Membranes and in Live Cells. Anal Chem 2015, 87, 5690-5697. [PubMed: 25927252]

34. Yeh H-C; Puleo CM; Ho Y-P; Bailey VJ; Lim TC; Liu K; Wang T-H Tunable Blinking Kinetics of Cy5 for Precise DNA Quantification and Single-Nucleotide Difference Detection. Biophys J 2008, 95, 729-737. [PubMed: 18424494]

35. Marquardt DW An Algorithm for Least-Squares Estimation of Nonlinear Parameters. SIAM J Appl Math 1963, 11, 431-441.

36. Ehrenberg M; Rigler R Rotational Brownian Motion and Fluorescence Intensify Fluctuations. Chemical Physics 1974, 4, 390-401.

37. Aragón SR; Pecora R Fluorescence Correlation Spectroscopy and Brownian Rotational Diffusion. Biopolymers 1975, 14, 119-137.

38. Kask P; Piksarv P; Pooga M; Mets Ü; Lippmaa E Separation of the Rotational Contribution in Fluorescence Correlation Experiments. Biophys J 1989, 55, 213-220. [PubMed: 19431738]

39. Widengren J; Mets Ü; Rigler R Photodynamic Properties of Green Fluorescent Proteins Investigated by Fluorescence Correlation Spectroscopy. Chemical Physics 1999, 250, 171-186.

40. Kapusta P Absolute Diffusion Coefficients: Compilation of Reference Data for FCS Calibration; Application Note, PicoQuant GmbH, 2010.

41. Yin Y; Yuan R; Zhao XS Amplitude of Relaxations in Fluorescence Correlation Spectroscopy for Fluorophores That Diffuse Together. J Phys Chem Lett 2013, 4, 304-309. [PubMed: 26283439]

42. Williams ATR; Winfield SA; Miller JN Relative Fluorescence Quantum Yields Using a ComputerControlled Luminescence Spectrometer. The Analyst 1983, 108, 1067-1071. 
43. Allen WA Measurement of Fluorescence Quantum Yields; Technical Note: 52019, Thermo Fisher Scientific, 2010.

44. Young RJ; Lovell PA; Introduction to Polymers, Third Edition; CRC Press: Boca Raton, FL, USA, 2011.

45. Zhao M; Jin L; Chen B; Ding Y; Ma H; Chen D Afterpulsing and Its Correction in Fluorescence Correlation Spectroscopy Experiments. Appl Opt 2003, 42, 4031-4036. [PubMed: 12868844]

46. Larson DR; Ow H; Vishwasrao HD; Heikal AA; Wiesner U; Webb WW Silica Nanoparticle Architecture Determines Radiative Properties of Encapsulated Fluorophores. Chem Mater 2008, 20, 2677-2684.

47. Cohen B; Martin C; Iyer SK; Wiesner U; Douhal A Single Dye Molecule Behavior in Fluorescent Core-Shell Silica Nanoparticles. Chem Mater 2012, 24, 361-372.

48. Iler RK The Chemistry of Silica: Solubility, Polymerizaion, Colloid and Surface Properties, and Biochemistry; Wiley: New York, USA, 1979.

49. Muddana HS; Morgan TT; Adair JH; Butler PJ Photophysics of Cy3-Encapsulated Calcium Phosphate Nanoparticles. Nano Lett 2009, 9, 1559-1566. [PubMed: 19260707]

50. Strickler SJ; Berg RA Relationship between Absorption Intensity and Fluorescence Lifetime of Molecules. J Chem Phys 1962, 37, 814-822.

51. Hirayama S; Phillips D Correction for Refractive Index in the Comparison of Radiative Lifetimes in Vapour and Solution Phases. J Photochemistry 1980, 12, 139-145.

52. Van Helden AK; Vrij A Contrast Variation in Light Scattering: Silica Spheres Dispersed in Apolar Solvent Mixtures. J Colloid Interface Sci 1980, 76, 418-433.

53. Mujumdar RB; Ernst LA; Mujumdar SR; Lewis CJ; Waggoner AS Cyanine Dye Labeling Reagents: Sulfoindocyanine Succinimidyl Esters. Bioconjugate Chem 1993, 4, 105-111. 

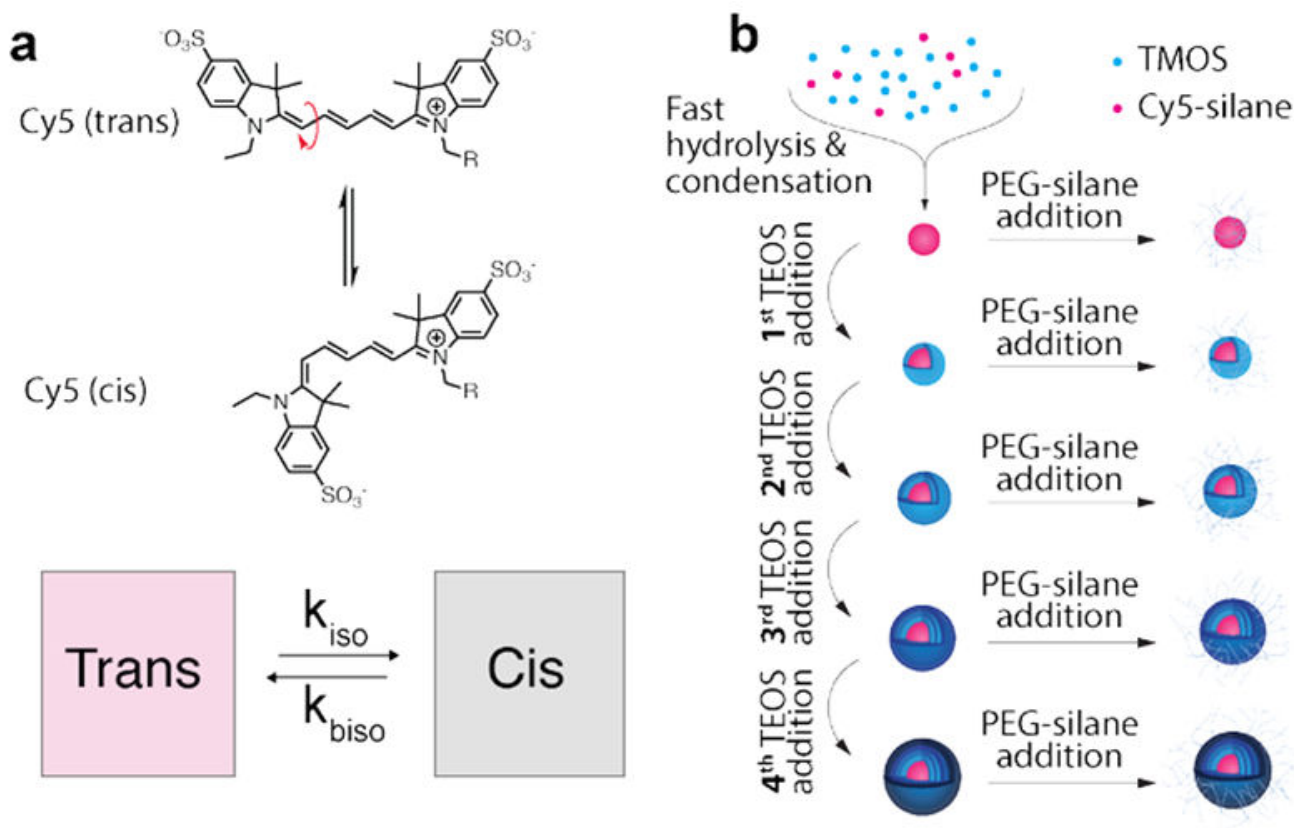

d
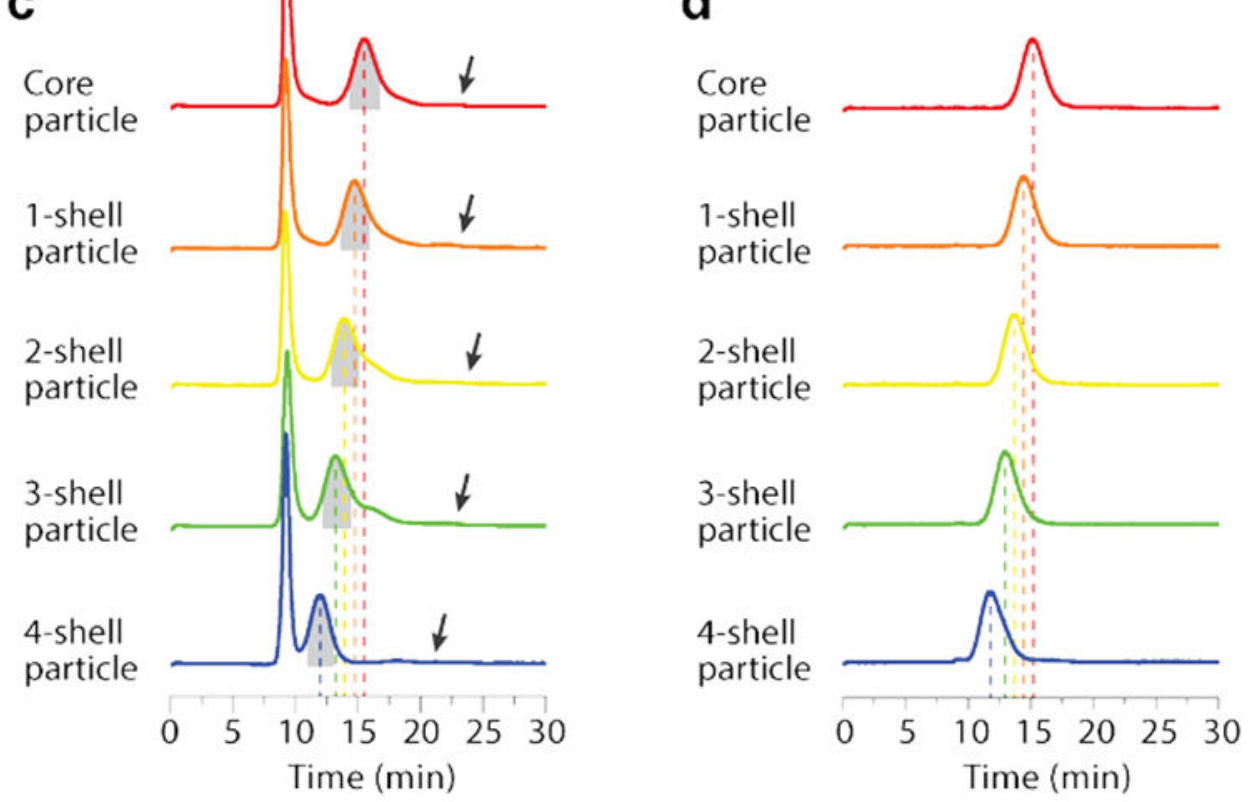

Figure 1. Dye details, particle synthesis, and particle characterization.

(a) Cy5 photo-induced cis-trans isomerization around the polymethinic chain, and corresponding kinetic model. (b) Schematic representation of step-wise synthesis of dyeencapsulating fluorescent core-shell SNPs. (c, d) GPC elugrams of as synthesized (c) and GPC purified (d) dye-encapsulating core-shell SNPs. 


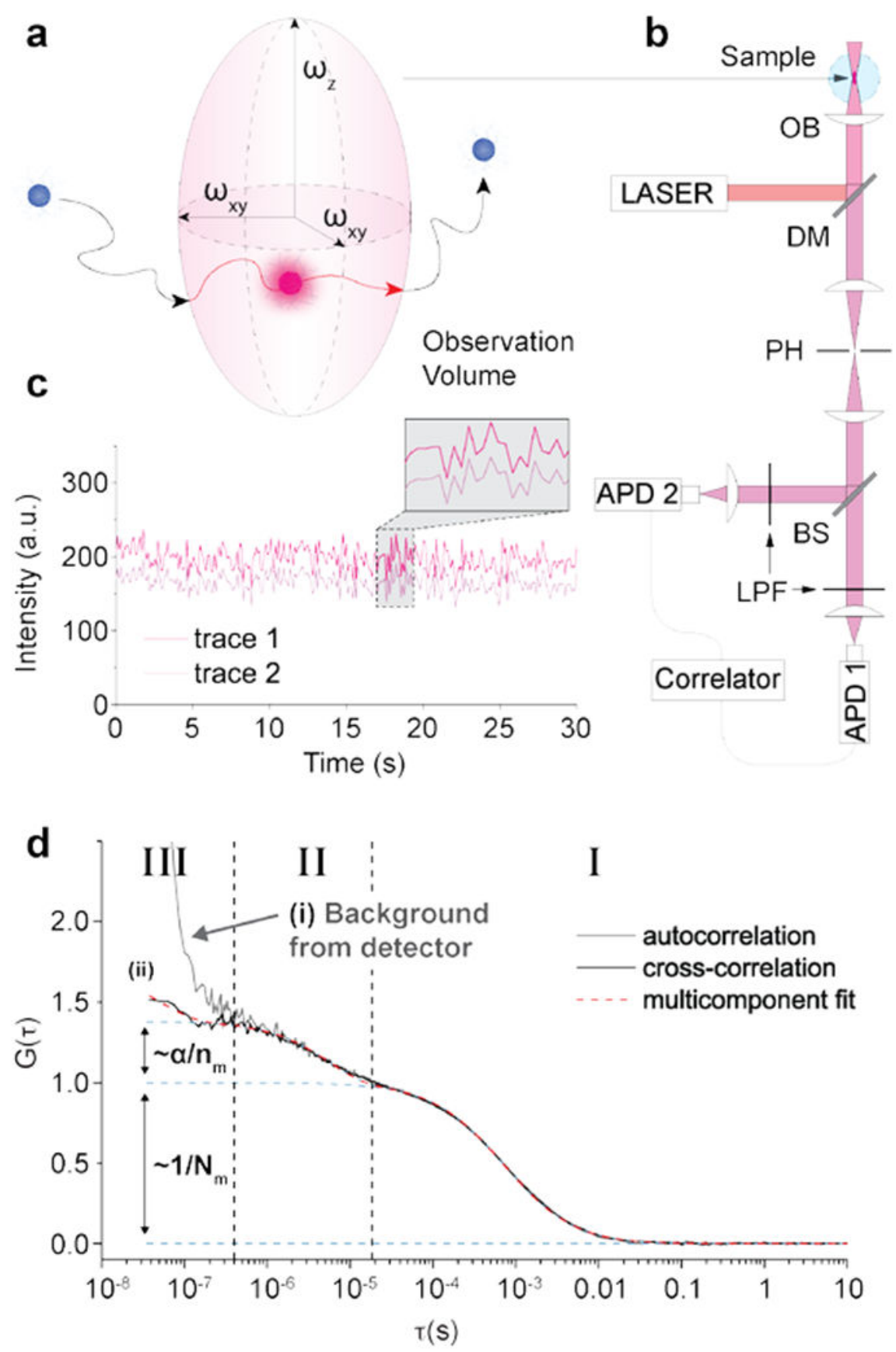

Figure 2. FSC schematic with effects of detector afterpulsing.

(a) Sample observation volume element of FCS laser beam with axial dimension, $\omega_{Z}$, radial dimensions, $\omega_{x y}$, and showing a fluorescent particle diffusing through. (b) Schematic of confocal FCS setup to remove effects of afterpulsing, depicting laser beam focal volume position, objective $(\mathrm{OB})$, laser, dichroic mirror $(\mathrm{DM})$, pinhole $(\mathrm{PH})$, beam splitter $(\mathrm{BS})$, long-pass filter (LPF), two avalanche photodiode detectors (APD $1 \&$ 2), and correlator. (c) Fluorescence traces as recorded by APD 1 and APD 2 from FCS measurement. Inset shows enlarged time window. (d) Characteristic FCS ACFs of fluorescent silica nanoparticles in 
this study: (i) with effects of afterpulsing (grey); with afterpulsing effects removed (black), and corresponding fit (red). Region I: translational diffusion; region II: photo-isomerization of cyanine dyes; and region III: high background from detector afterpulsing (i) or particle rotational diffusion (ii). 

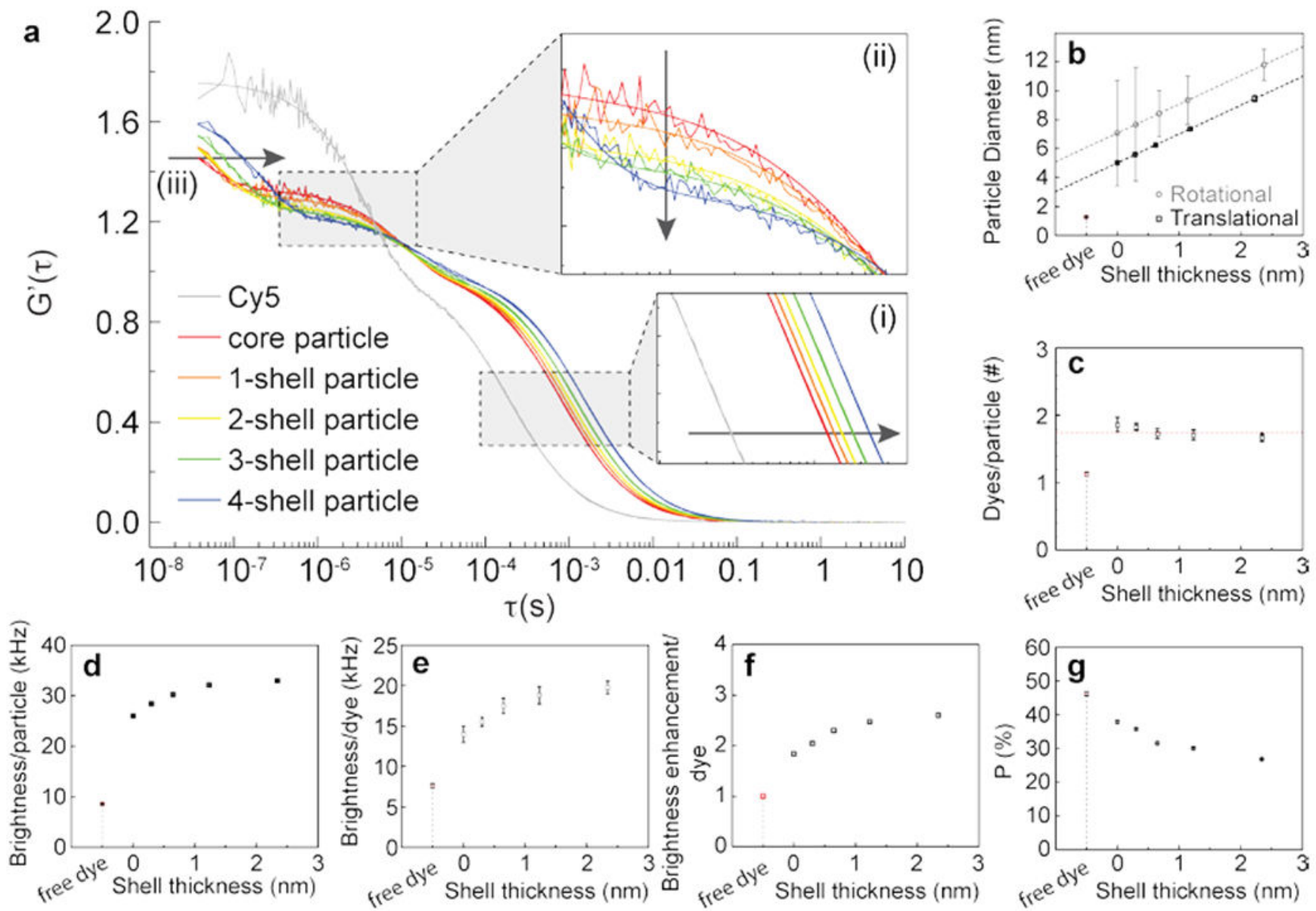

Figure 3. FCS measurements and associated analyses.

(a) Comparison of FCS results (with afterpulsing effects removed) for pure Cy5 (grey) and Cy5-encapsulating core-shell SNPs (colored) together with associated fits. (b) Particle diameter as a function of silica shell thickness as determined by translational diffusion (black) versus rotational diffusion (grey). (c) Dyes per particle as determined by a combination of FCS and absorption spectroscopy. (d) Brightness per particle as a function of silica shell thickness. (e) Brightness per encapsulated dye as a function of silica shell thickness. (f) Brightness enhancement per dye as a function of silica shell thickness. (g) Percentage of photoisomerizing dyes as determined by FCS as a function of silica shell thickness. 

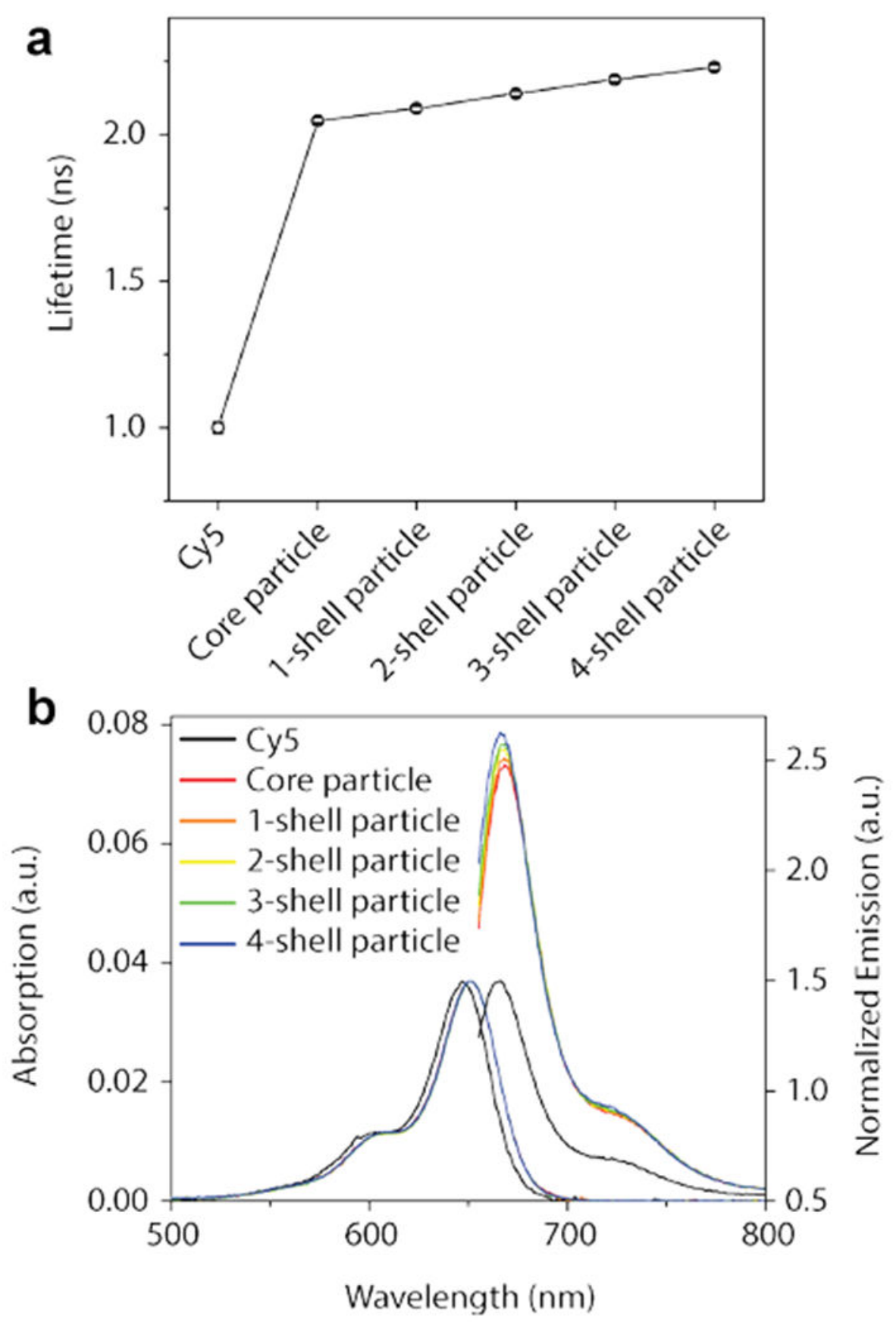

Figure 4. Fluorescence lifetimes and steady-state absorption and emission.

(a) Comparison of fluorescence lifetimes of Cy5-encapsulating core-shell SNPs versus free Cy5 dye. (b) Intensity-matched absorption spectra of free Cy5 dye and Cy5-encapsulating fluorescent core-shell SNPs together with corresponding emission spectra. Emission spectra are normalized with respect to $\mathrm{Cy} 5$ in water. 
Table 1.

Fraction of Cy5 molecules being in the non-fluorescent cis conformation, $\mathrm{P}$, relaxation time of Cy5 cis-trans isomerization, $\tau_{P}$, effective rate of isomerization from trans to cis, $k_{i s o}$, effective rate of back-isomerization form cis to trans, $k_{b i s o}$, equilibrium constant of back-isomerization, $K$. Normalized values are with respect to Cy5 in water at $20^{\circ} \mathrm{C}$.

\begin{tabular}{lccccccc}
\hline Shell thickness & $\mathbf{P}[\%]$ & $\boldsymbol{\tau}_{\mathrm{P}}[\boldsymbol{\mu s}]$ & $\boldsymbol{k}_{\text {iso }}\left[\boldsymbol{\mu s}^{-\mathbf{1}}\right]$ & $\boldsymbol{k}_{\text {iso }}($ normalized $)$ & $\boldsymbol{k}_{\text {biso }}\left[\boldsymbol{\mu \mathbf { s } ^ { - 1 } ]}\right.$ & $\boldsymbol{k}_{\text {biso }}(\mathbf{n o r m a l i z e d})$ & $\mathbf{k}$ \\
\hline $\mathbf{C y 5}$ & $46.2 \pm 0.6$ & $3.9 \pm 0.1$ & $0.118 \pm 0.003$ & $1.00 \pm 0.03$ & $0.137 \pm 0.006$ & $1.00 \pm 0.06$ & $1.16 \pm 0.06$ \\
$\mathbf{0 ~} \mathbf{~ n m}$ & $37.9 \pm 0.3$ & $10.6 \pm 0.5$ & $0.036 \pm 0.002$ & $0.30 \pm 0.02$ & $0.058 \pm 0.005$ & $0.43 \pm 0.04$ & $1.64 \pm 0.15$ \\
$\mathbf{0 . 3} \mathbf{~ n m}$ & $35.8 \pm 0.3$ & $11.2 \pm 0.1$ & $0.032 \pm 0.001$ & $0.27 \pm 0.01$ & $0.057 \pm 0.001$ & $0.42 \pm 0.02$ & $1.80 \pm 0.04$ \\
$\mathbf{0 . 7} \mathbf{~ n m}$ & $31.6 \pm 0.3$ & $12.0 \pm 0.8$ & $0.026 \pm 0.002$ & $0.22 \pm 0.02$ & $0.057 \pm 0.006$ & $0.42 \pm 0.05$ & $2.17 \pm 0.27$ \\
$\mathbf{1 . 2} \mathbf{~ n m}$ & $30.1 \pm 0.3$ & $13.1 \pm 0.6$ & $0.023 \pm 0.001$ & $0.20 \pm 0.01$ & $0.054 \pm 0.003$ & $0.39 \pm 0.03$ & $2.32 \pm 0.18$ \\
$\mathbf{2 . 3} \mathbf{~ n m}$ & $26.8 \pm 0.2$ & $17.7 \pm 0.7$ & $0.015 \pm 0.001$ & $0.13 \pm 0.01$ & $0.041 \pm 0.002$ & $0.30 \pm 0.02$ & $2.73 \pm 0.18$ \\
\hline
\end{tabular}


Table 2.

Quantum yield as determined by FCS, $\varphi_{F}$, fluorescence lifetime, $\tau_{F}$, radiative rate, $k_{F}$, and non-radiative rate, $k_{n r}$ Normalized values are with respect to Cy5 in water.

\begin{tabular}{lcccccc}
\hline Shell thickness & $\boldsymbol{\varphi}_{\boldsymbol{F}}[\%](\mathbf{F C S})$ & $\boldsymbol{\tau}_{\boldsymbol{F}}[\mathbf{n s}]$ & $\boldsymbol{\kappa}_{r}\left[\mathbf{n s}^{-\mathbf{1}}\right]$ (FCS) & $\boldsymbol{\kappa}_{r}[$ normalised $](\mathbf{F C S})$ & $\boldsymbol{\kappa}_{n r}\left[\mathbf{n s}^{-\mathbf{1}}\right](\mathbf{F C S})$ & $\boldsymbol{\kappa}_{n r}[$ normalised $](\mathbf{F C S})$ \\
\hline $\mathbf{C y 5}$ & $0.20^{a}$ & 1.00 & 0.20 & 1.00 & 0.80 & 1.00 \\
$\mathbf{0 ~} \mathbf{~ m}$ & 0.37 & 2.05 & 0.18 & 0.90 & 0.31 & 0.39 \\
$\mathbf{0 . 3} \mathbf{~ m m}$ & 0.41 & 2.09 & 0.20 & 0.98 & 0.28 & 0.35 \\
$\mathbf{0 . 7} \mathbf{~ n m}$ & 0.46 & 2.14 & 0.21 & 1.07 & 0.25 & 0.31 \\
$\mathbf{1 . 2} \mathbf{~ n m}$ & 0.49 & 2.19 & 0.23 & 1.13 & 0.23 & 0.29 \\
$\mathbf{2 . 3} \mathbf{~ n m}$ & 0.52 & 2.23 & 0.23 & 1.16 & 0.22 & 0.27 \\
\hline
\end{tabular}

${ }^{a}$ Quantum yield from ref. 53. 
Table 3.

Quantum yield as determined by steady-state absorption and emission spectroscopy, $\Phi_{F}$, fluorescence lifetime, $\tau_{F}$, radiative rate, $k_{r}$, and non-radiative rate, $k_{n r}$. For easy comparison, the quantum yield as obtained from FCS, $\varphi_{F}$, and the fraction of Cy5 molecules being in the non-fluorescent cis conformation, $\mathrm{P}$, are relisted. Normalized values are with respect to Cy5 in water.

\begin{tabular}{lccccccc}
\hline Shell thickness & $\phi_{F}[\%]$ & $\boldsymbol{\kappa}_{r}\left[\mathbf{n s}^{-1}\right]$ & $\boldsymbol{\kappa}_{r}($ normalized $)$ & $\boldsymbol{\kappa}_{n r}\left[\mathrm{~ns}^{-1}\right]$ & $\boldsymbol{\kappa}_{n r}($ normalized $)$ & $\boldsymbol{\phi}_{F}[\%](\mathbf{F C S})$ & $\mathbf{P}[\%](\mathbf{F C S})$ \\
\hline $\mathbf{C y 5}$ & $0.20^{a}$ & 0.20 & 1.00 & 0.80 & 1.00 & $0.20^{a}$ & $46.2 \pm 0.6$ \\
$\mathbf{0 ~} \mathbf{~ m}$ & 0.48 & 0.23 & 1.17 & 0.25 & 0.32 & 0.37 & $37.9 \pm 0.3$ \\
$\mathbf{0 . 3} \mathbf{~ m m}$ & 0.49 & 0.24 & 1.18 & 0.24 & 0.30 & 0.41 & $35.8 \pm 0.3$ \\
$\mathbf{0 . 7} \mathbf{~ n m}$ & 0.50 & 0.23 & 1.17 & 0.23 & 0.29 & 0.46 & $31.6 \pm 0.3$ \\
$\mathbf{1 . 2} \mathbf{~ n m}$ & 0.51 & 0.23 & 1.17 & 0.22 & 0.28 & 0.49 & $30.1 \pm 0.3$ \\
$\mathbf{2 . 3} \mathbf{~ n m}$ & 0.54 & 0.24 & 1.20 & 0.21 & 0.26 & 0.52 & $26.8 \pm 0.2$ \\
\hline
\end{tabular}

${ }^{a}$ Quantum yield from ref. 53. 